\title{
DISTRIBUTION OPTIMALE DU COURANT DANS UNE BOBINE SANS FER POUR MESURES DE SUSCEPTIBILITÉ MAGNÉTIQUE
}

\author{
R. BLANCHET $(*)$, F. GAUME et A. BONDEAU \\ Physique Expérimentale, Université Claude-Bernard, Lyon I \\ 43, boulevard du 11-Novembre-1968, 69-Villeurbanne
}

(Reçu le 15 octobre 1972)

\begin{abstract}
Résumé. - Pour obtenir des valeurs à la fois intenses et uniformes du produit du champ magnétique par son gradient axial, on étudie des bobines à disques en imposant, en plus de la variation radiale, une variation axiale de la densité de courant, qui améliore de $10 \%$ le rapport de la force magnétique à la puissance consommée. Une méthode systématique, susceptible d'autres applications, est mise au point pour l'optimisation de cette répartition du courant. Par un procédé diminuant les résistances de contact, on a construit une bobine maquette, qui montre les avantages de ce dispositif par rapport aux montages classiques.
\end{abstract}

\begin{abstract}
In order to get both strong and uniform values of the product of the magnetic field and its axial gradient, disk magnet-coils have been studied in causing an axial variation in addition of the radial variation of the current density. The ratio of the magnetic strength to the consumed power is improved by $10 \%$. A systematic method, which could be useful in other cases, is studied for the optimization of this distribution of current. Thanks to a process which reduces the resistances of contact, a proof coil has been designed, showing the advantages of this apparatus compared to the usual instruments.
\end{abstract}

1. Introduction. - Un dispositif de mesure de susceptibilité magnétique est caractérisé en premier lieu par la valeur du paramètre $F=H(\partial H / \partial a)$, produit du champ magnétique en un point repéré par une coordonnée $a$, par le gradient du champ au même point et dans la direction de cette coordonnée. La force à laquelle est soumis un échantillon étant proportionnelle à $F$, une valeur élevée de ce produit augmente la sensibilité de l'appareil, ou la précision des mesures.

En second lieu, l'uniformité de $F$ dans un volume $V$ supérieur à celui de l'échantillon améliore la fidélité, facilite les enregistrements automatiques en évitant une localisation rigoureuse de l'échantillon et autorise un accroissement de la masse utilisée, donc aussi de la force magnétique.

Il est difficile de satisfaire simultanément ces deux conditions de valeur élevée et d'uniformité en employant un électroaimant à circuit ferromagnétique. Il est cependant possible d'uniformiser $H(\partial H / \partial a)$ au voisinage d'une portion de plan, en donnant une forme appropriée aux pièces polaires [1]. En revanche, on peut obtenir des valeurs plus élevées de $F$. $V$ en utilisant des bobines sans fer fonctionnant à la température ordinaire, et en calculant la répartition optimale du courant dans ces bobines.

Dans ce cas, la coordonnée $a$ est prise le long de l'axe de l'enroulement, axe dont l'origine $O$ corres-

$\left(^{*}\right)$ R. Blanchet, actuellement à l'Ecole Centrale de Lyon. pond au point où l'on désire réaliser une valeur élevée et uniforme de $F$. Pour des raisons de simplicité de construction et de robustesse, les enroulements envisagés sont des bobines massives à gorges rectangulaires (Fig. 1), de rayons intérieur $y_{1}$ et extérieur $y_{2}$. Une spire, de section $\mathrm{d} x \mathrm{~d} y$, est caractérisée par son abscisse $x$, son rayon $y$, et la densité $i$ du courant qui la parcourt.

Comme pour l'étude d'autres effets magnétiques [2], [3], [4], trois dispositifs, dont les deux premiers ont été étudiés précédemment [5], peuvent être envisagés.

1. Deux bobines ordinaires, à densités de courant uniformes, $i$ et $i^{\prime}$.

2. Deux bobines à disques de Bitter, de mêmes rayons. Leurs densités de courant sont alors :

$$
i=i_{1} \frac{y_{1}}{y} ; \quad i^{\prime}=i_{1}^{\prime} \frac{y_{1}}{y},
$$

$i_{1}$ et $i_{1}^{\prime}$ désignant les valeurs prises par la densité de courant sur le bord intérieur des disques des deux bobines.

3. Une bobine à densité de courant variable suivant l'axe [6], [7], donnée par une loi de distribution :

$$
i=i_{01} \frac{y_{1}}{y} f(x),
$$

où $i_{01}$ est la valeur de cette densité pour $x=0$ et $y=y_{1}$. 


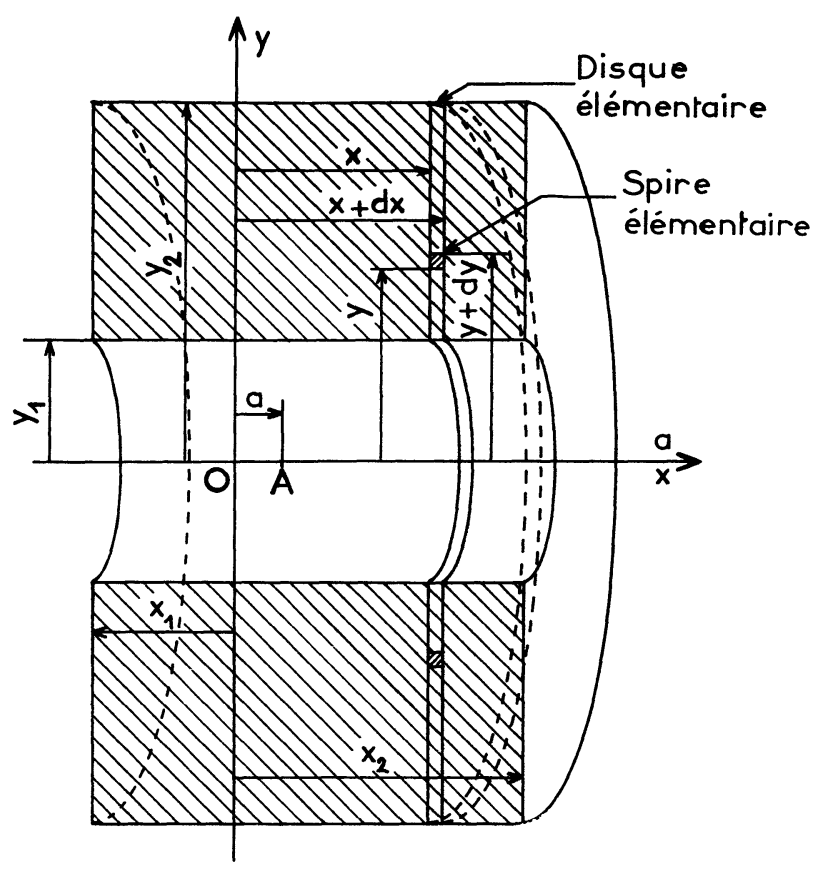

FIG. 1. - Coupe longitudinale d'une bobine massive.

Le second dispositif donne, par rapport au premier, une amélioration de $27,5 \%$ du rapport $F / P$ de l'effet magnétique recherché à la puissance consommée, pour une même valeur du coefficient de foisonnement $\eta$ et une uniformité identique [5]. Par analogie avec les calculs de G. Jaillet et R. Pourraz [2], recherchant des valeurs élevées de $H(\partial H / \partial a)$ sans condition spéciale d'uniformité, on peut encore prévoir une amélioration de l'ordre de $10 \%$ en passant du deuxième au troisième dispositif. Le problème est donc de déterminer et de réaliser au mieux la fonction $f(x)$, en recherchant une valeur maximale de $F$ pour une puissance $P$ donnée [8].

2. Recherche de la répartition axiale $f(x)$ de la densité de courant. - En désignant par $x_{1}$ et $x_{2}$ les abscisses des faces terminales de la bobine, le produit $F$ et la puissance $P$ ont respectivement pour expression :

$$
\begin{aligned}
F & =H \frac{\partial H}{\partial a} \\
& =i_{01}^{2}\left(\frac{y_{1}}{\eta}\right)^{2} \int_{x_{1}}^{x_{2}} f(x) \varphi(x, a) \mathrm{d} x \int_{x_{1}}^{x_{2}} f(x) \frac{\partial \varphi(x, a)}{\partial a} \mathrm{~d} x,
\end{aligned}
$$

avec :

$$
\begin{aligned}
\varphi(x, a) & =\frac{\partial H}{\partial x} \frac{\eta}{i_{01} y_{1} f(x)} \\
& =\frac{1}{2}\left\{\left[y_{1}^{2}+(x-a)^{2}\right]^{-1 / 2}-\left[y_{2}^{2}+(x-a)^{2}\right]^{-1 / 2}\right\}
\end{aligned}
$$

et

$$
P=2 \pi \rho i_{01}^{2} y_{1}^{2} \frac{1}{\eta} \ln \frac{y_{2}}{y_{1}} \int_{x_{1}}^{x_{2}}[f(x)]^{2} \mathrm{~d} x,
$$

$\rho$ étant la résistivité du cuivre.
La répartition du courant dans le second dispositif (association de deux bobines de Bitter) peut aussi bien être décrite dans le cadre du troisième cas, $f(x)$ étant alors une fonction créneau à deux paliers, représentée en valeurs relatives $\left(x_{\mathrm{r}}=x / y_{1}\right)$ sur la figure $2 a$. Ce sera la première étape, ou distribution $f_{1}(x)$, dans l'élaboration de $f(x)$ optimale. Le principe du calcul est alors de transformer progressivement cette répartition en une fonction escalier aux paliers de plus en plus nombreux et étroits afin de rapprocher $f(x)$, sans altération notable de l'uniformité de $F$, de la fonction continue idéale qui correspondrait au maximum du rapport $F / P$. Après chaque modification de $f(x)$, le tracé de la courbe $F / F_{0}=g\left(a_{\mathrm{r}}\right)$ et le calcul de $P$ permettent de vérifier le maintien de l'uniformité de $F$ et l'accroissement de $F / P$.

2.1 DÉTERMINATION APPROCHÉE DE LA FONCTION $f(x)$. - Au début, l'intuition suffit pour guider les modifications apportées à $f_{1}(x)$. Plusieurs essais conduisent à une répartition nouvelle $f_{2}(x)$ (soit $f_{2}\left(x_{\mathrm{r}}\right)$ sur la figure $2 a)$. La courbe $F / F_{0}$ correspondante,

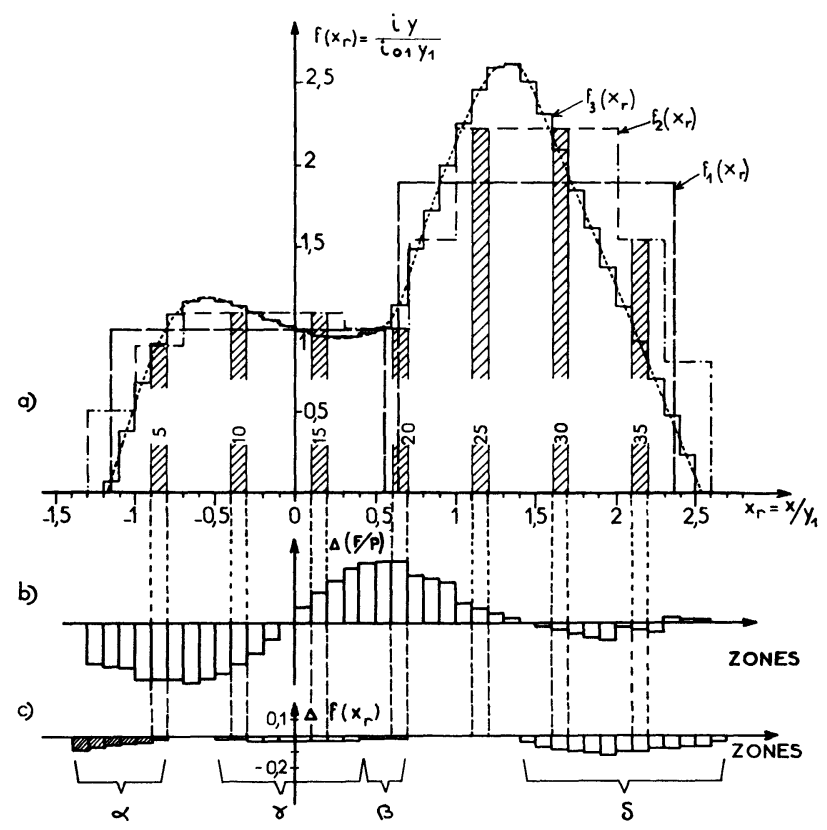

FIG. 2. - $a$ ) Répartitions successives de la densité de courant. b) Variations de $F / P$ (unités arbitraires) suivant la zone dans laquelle on modifie la densité de courant. c) Association de deux paires d'interventions $(\alpha, \beta)$ et $(\gamma, \delta)$ sur la fonction $f(x)$, sans effet sur l'uniformité de $F=H(\partial H / \partial a)$ mais faisant croître $F / P$.

$g_{2}\left(a_{\mathrm{r}}\right)$ en valeurs relatives (Fig. 3) montre que l'uniformité est très satisfaisante. Le calcul de $P$ révèle par ailleurs une économie de puissance de 5,2\% par rapport à la distribution $f_{1}(x)$ à deux paliers, pour un effet magnétique égal. Les calculs numériques sont effectués avec un rapport $y_{2} / y_{1}=3$.

2.2 AfFinement SystématiQue DE LA FONCTION $f(x)$. - Pour améliorer la fonction $f_{2}(x)$, on a le choix entre la programmation de l'ensemble du problème, 


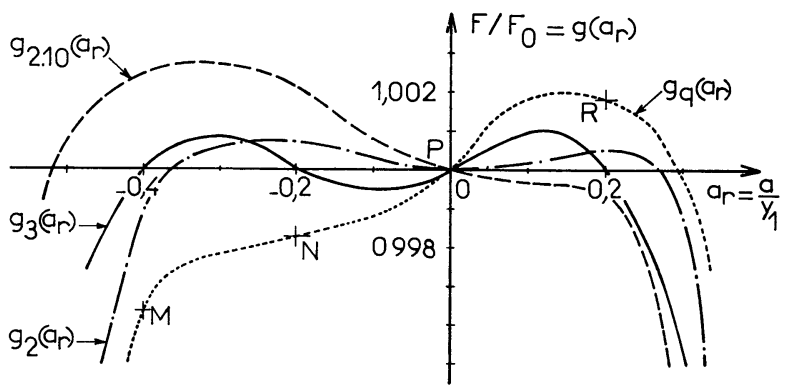

FIG. 3. - Variations relatives de $F$ (proportionnel à la force magnétique) le long de l'axe de la bobine. $g_{2}$ et $g_{3}$ : conséquences des distributions $f_{2}$ et $f_{3}$ de la densité de courant. $g_{2.10}$ : résultat d'une modification locale de $f_{2}$ (dixième zone). $g_{a}$ : courbe quelconque illustrant la méthode d'uniformisation.

et la méthode exposée ci-dessous. Cette dernière solution a été préférée au calcul exclusivement itératif à l'ordinateur, car elle permet de diriger la progression de $f(x)$ vers sa forme optimale, par l'examen détaillé des effets de modifications locales élémentaires apportées à cette fonction.

2.2.1 Effets des déformations élémentaires imposées $\grave{a} f_{2}(x)$. - Le graphique de $f_{2}\left(x_{\mathrm{r}}\right)$ est partagé en zones de largeur $\delta x_{\mathrm{r}}=0,1$ (Fig. 2a). Dans chacune de ces zones, on augmente ensuite $i$ d'une valeur constante et on détermine chaque fois la courbe $g\left(a_{\mathrm{r}}\right)$ correspondante, ainsi que le rapport $F / P$. Une courbe de ce type accompagne $g_{2}\left(a_{\mathrm{r}}\right)$ sur la figure 3 , à titre d'exemple. On trace également (Fig. $2 b$ ), la modification du rapport $F / P$ suivant la zone affectée.

L'examen de ces graphiques et l'utilisation des tableaux numériques correspondants permet de prévoir l'effet d'une modification quelconque de $f\left(x_{\mathrm{r}}\right)$, si son amplitude est assez petite. On la considérera en effet comme une perturbation élémentaire produisant des effets qui lui sont proportionnels.

2.2.2 Amélioration systématique de l'uniformité. Une modification du tracé de $f\left(x_{\mathbf{r}}\right)$, par passage à un nombre supérieur de paliers, altère en général l'uniformité de $F$. Pour rétablir cette uniformité, on cherche à placer avec une précision donnée, sur l'axe $O a_{\mathrm{r}}$, les trois points $\mathrm{M}, \mathrm{N}$ et $\mathrm{R}$ de $g_{\mathrm{q}}\left(a_{\mathrm{r}}\right)$, courbe quelconque à uniformiser (Fig. 3). Or les résultats des déformations élémentaires (Fig. 3), montrent qu'il est difficile, en agissant sur $f(x)$ dans une seule zone (Fig. 2a), de rapprocher l'un des trois points de l'axe $O a_{\mathrm{r}}$ sans en éloigner un autre. On effectue donc ces modifications par paires, avec des amplitudes telles que l'altération simultanée des deux zones maintienne stationnaires deux des trois points $\mathbf{M}, \mathbf{N}$ et $\mathbf{R}$. Quatre couples de modifications remplissant ces conditions pour $\mathrm{M}$ et $\mathrm{N}$ sont d'abord établis. On détermine alors leurs influences respectives sur $F / P$ ainsi que sur l'ordonnée du troisième point $R$. La même opération est ensuite effectuée en gardant cette fois $\mathrm{N}$ et $\mathrm{R}$ stationnaires.

2.2.3 Accroissement de $F$ sans altération de l'uniformité. - $\mathrm{Au}$ cours des opérations précédentes concernant l'uniformité, on utilise les couples de modi- fications de $f\left(x_{\mathrm{r}}\right)$ qui augmentent le plus le rapport $F / P$. Une distribution nouvelle ainsi déterminée donne une uniformité de $H(\partial H / \partial a)$ à $0,1 \%$ près, tout en réalisant une économie de puissance de 7,3\% par rapport aux performances de la répartition $f_{1}(x)$ à deux paliers [5].

Il est possible d'aller plus loin. En effet, dans la série des couples de modifications de $f\left(x_{\mathrm{r}}\right)$ laissant deux points de $g\left(a_{\mathrm{r}}\right)$ stationnaires, $\mathrm{N}$ et $\mathrm{R}$ par exemple, on peut en trouver deux qui remplissent la condition suivante : ils ont des effets inverses sur l'ordonnée $\mathrm{du}$ point $\mathrm{M}$ et provoquent l'un et l'autre une économie de puissance. La figure $2 c$ représente un exemple d'une telle association de deux paires d'interventions sur $f\left(x_{\mathrm{r}}\right)$. Les modifications sont réparties sur les zones voisines des zones caractéristiques pour garder à $f\left(x_{\mathrm{r}}\right)$ un aspect de continuité. Ce genre d'opération permet donc d'augmenter $F / P$ sans altérer l'uniformité. En la répétant plusieurs fois, on arrive à une distribution $f_{3}(x)$ (Fig. 2a). La courbe correspondante $g_{3}\left(a_{\mathrm{r}}\right)$ (Fig. 3) met en évidence une uniformité à $0,1 \%$ pour $-0,4 \leqslant a_{\mathrm{r}} \leqslant 0,2$, voisine de celle de la courbe $g_{2}$, cependant que l'accroissement de $F / P$ par rapport à la répartition $f_{1}(x)$ est maintenant de $10 \%$ au lieu de $5,2 \%$.

La méthode systématique utilisée ici pour accroître la valeur et l'uniformité de $F$ pourrait s'appliquer à d'autres problèmes d'optimisation.

3. Réalisation pratique de la répartition $f(x)$. - La réalisation exacte de la fonction continue équivalente à $f_{3}(x)$ (tracé pointillé, Fig. $2 a$ ) exigerait l'usinage d'une hélice à pas variable. On obtient une bonne approximation de cette hélice en groupant en parallèle un nombre variable $n$ de disques de cuivre de $0,1 \mathrm{~mm}$ et $0,2 \mathrm{~mm}$ d'épaisseur pour former des spires qui, isolées par des disques en presspahn, sont ensuite connectées en série. Le nombre $n$ est déterminé par le fait qu'une spire d'abscisse moyenne $x$ doit avoir une épaisseur inversement proportionnelle à $f_{3}(x)$.

Le groupement des disques est fait selon une nouvelle disposition, qui permet une diminution de la résistance de contact par rapport à une méthode précédemment utilisée [3], [7], elle-même dérivée de celle de Bitter [9]. La figure 4 montre comment les spires sont constituées de $n$ disques accolés, décalés en hélice les uns par rapport aux autres et liés entre eux par une soudure à l'étain sur la tranche extérieure, ce qui facilite leur montage. De cette manière les résistances de contact dans une spire donnée sont en parallèle au lieu d'être en série [3]. On est également amené à fabriquer des fractions de spires, les disques étant alors découpés suivant l'angle correspondant à cette fraction. Ce mode d'enroulement amènerait sans doute une amélioration dans la construction de bobines sans fer destinées à d'autres usages, et même dans le montage de secondaires de transformateurs basse tension.

La bobine ainsi construite (Fig. 5) comporte deux enroulements coaxiaux connectés en série. Le refroi- 


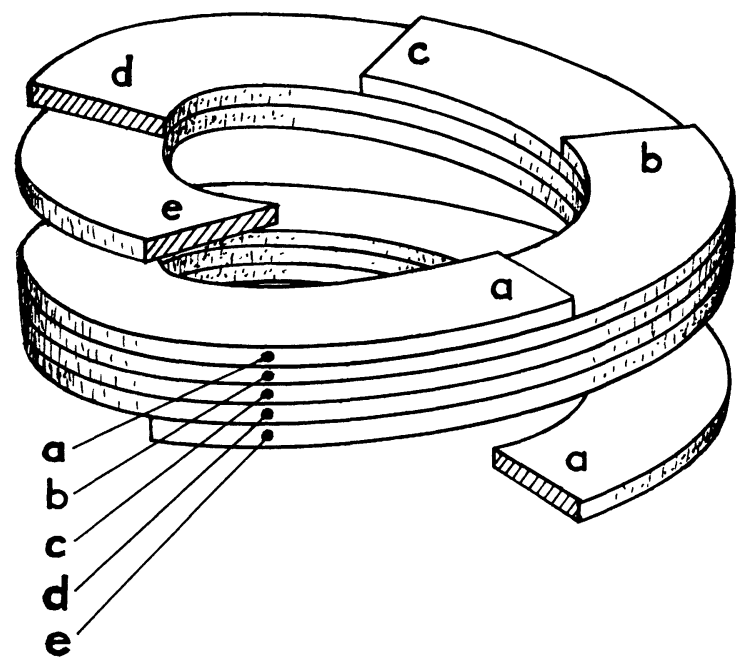

FIG. 4. - Exemple de spire hélicoïdale comprenant cinq disques connectés en parallèle, fendus chacun suivant un rayon et déformés hélicoïdalement.

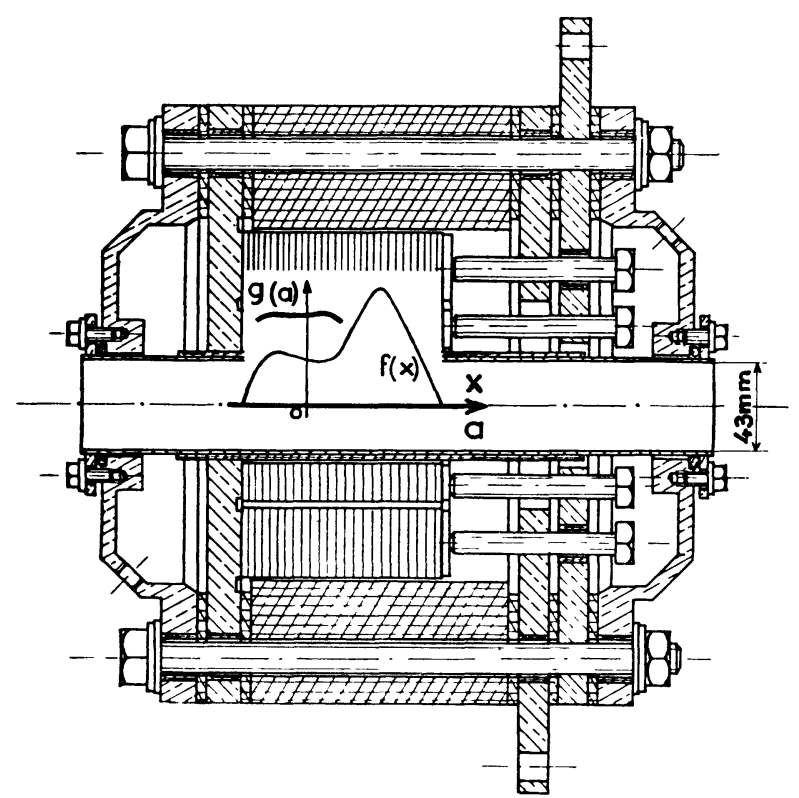

FIG. 5. - Coupe longitudinale de la bobine et variations de $f(x)$ et $g(a)$.

dissement est assuré par une circulation d'huile et un échangeur huile-eau. L'étude préalable de ce refroidissement fait prévoir, avec un coefficient d'échange thermique superficiel de l'ordre de $1000 \mathrm{~W} / \mathrm{m}^{2} .{ }^{\circ} \mathrm{C}$, une puissance maximale de $1380 \mathrm{~W}$. Cette valeur est déterminée en imposant à la température du cuivre de ne dépasser en aucun point $105^{\circ} \mathrm{C}$, limite admissible pour le presspahn imbibé d'huile.

Les spires étant refroidies seulement par leurs tranches intérieures et extérieures, la température n'est pas uniforme le long d'un rayon. La variation maximale calculée n'est cependant que de $1,8^{\circ} \mathrm{C}$, ce qui ne fausse pas notablement la répartition radiale du courant.

4. Essais et résultats. - La maquette construite a été étalonnée à l'aide d'un échantillon paramagnétique de susceptibilité connue, et d'une balance au $1 / 100$ de mg. Les courbes $g(a)$ expérimentale et théorique sont représentées sur la figure 6. On obtient, pour $y_{1}=2,5 \mathrm{~cm}, H(\partial H / \partial a)$ stationnaire à moins de $1 \%$ près dans un volume cylindrique de $2,5 \mathrm{~cm}$ de diamètre et $2 \mathrm{~cm}$ de long, soit environ $10 \mathrm{~cm}^{3}$. Ce volume est situé dans un canal libre de $43 \mathrm{~mm}$ de diamètre, ce qui le rend accessible à des dispositifs tels que cryostat ou four.

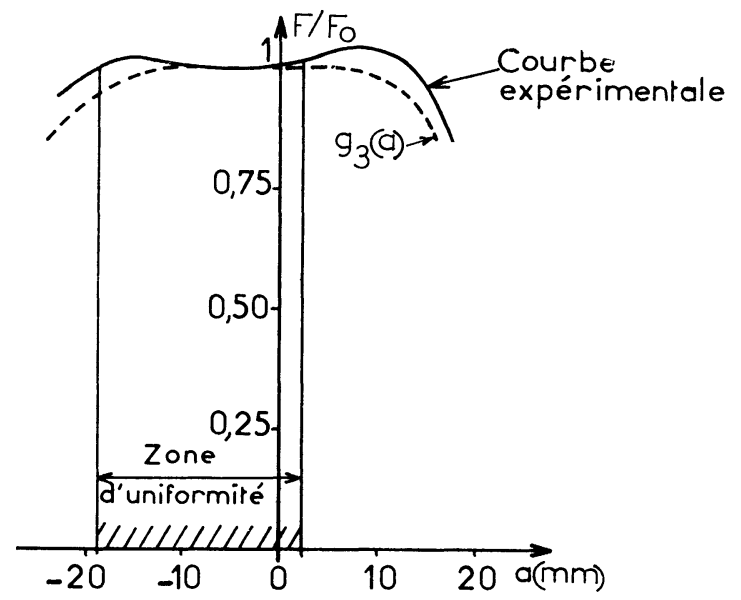

FIG. 6. - Courbes $g(a)$ expérimentale et théorique pour $y_{1}=25 \mathrm{~mm}$.

Les essais d'échauffement confirment assez bien les prévisions. On peut, tout en gardant une marge de sécurité, adopter une intensité maximale de $160 \mathrm{~A}$, ce qui correspond à une puissance de $1150 \mathrm{~W}$, et à $H \frac{\partial H}{\partial a} \approx 0,36 \times 10^{12} \mathrm{~A}^{2} / \mathrm{m}^{3}=0,57 \times 10^{6} \mathrm{Oe}^{2} / \mathrm{cm}$.

Quant au produit de ce paramètre par le volume dans lequel on l'obtient avec l'uniformité voulue, il atteint

$$
V \cdot H \frac{\partial H}{\partial a} \approx 3,6 \times 10^{6} \mathrm{~A}^{2}=5,7 \times 10^{6} \mathrm{Oe}^{2} . \mathrm{cm}^{2},
$$

soit environ six fois plus que ce que l'on obtient couramment avec les électroaimants pour mesures de susceptibilité magnétique.

Des améliorations complémentaires peuvent être envisagées d'une part sur le plan du calcul par une détermination de la valeur optimale de $y_{2} / y_{1}$, rapport des rayons des disques, et d'autre part sur le plan pratique par une construction mieux refroidie, à disques perforés.

De plus, si l'on multiplie toutes les dimensions de l'enroulement par un facteur $k$, tout en maintenant les conditions d'un bon refroidissement, les relations de similitudes concernant ce problème [6] montrent que les valeurs limites de $P, F$ et $F . V$ sont multtpliées respectivement par $k^{3}, k$ et $k^{4}$. Un accroissement des dimensions de la bobine d'essai présenterait donc un intérêt certain, surtout en ce qui concerne le produit $V . H(\partial H / \partial a)$. 


\section{Bibliographie}

[1] Develey G. et Rimet G., Formes de pièces polaires d'électroaimant pour mesures de susceptibilités paramagnétiques. J. Phys. (Phys. Appl.) 24 (1963) 137A.

[2] Gaume F., Cancade J. C., Jaillet G., Pourraz R. et Berthier P., Les champs magnétiques intenses. Colloque international du CNRS, Grenoble 1966. Editions du CNRS, Paris 1967, p. 149.

[3] Bondeau A., Blanchard P. et Gaume F., Production d'un champ magnétique de topographie adaptée aux expériences de magnéto-optique. $R G E 77$ (1968) 917.

[4] Gaume F., Bondeau A. et Boyrivent A., New Solenoid Magnets. Intern. Conf. on High Magnetic Fields, MIT Cambridge 39 (Mass.), USA, 1961. (Erratum : p. 35 , tabl. 2 , col. 5 , en bas, lire $392 \times 10^{3}$ au lieu de $357 \times 10^{3}$.)
[5] Eymard M. F., Gaume F. et Blanchet R., C. $R$. Acad. Sci. B, Paris 274 (1972) 1079-82.

[6] Gaume F., Bobines sans fer pour la production de champs magnétiques permanents très intenses. Calcul et réalisation. Thèse, $1^{\mathrm{er}}$ juin 1956, Paris. Journal des recherches du CNRS, Vol. 9, $\mathrm{n}^{\circ} 43$, p. $93-115 ; n^{\circ} 44$, p. $247-271 ; n^{\circ} 45$, p. $287-303$.

[7] Gaume F., Nouvelles bobines sans fer pour la production de champs magnétiques permanents ou transitoires. Brevet CNRS No 1209 196, Paris.

[8] Blanchet R., Bobine électromagnétique sans fer destinée à la mesure de susceptibilités magnétiques. Thèse CNAM, Lyon (1969).

[9] BITTER F., Rev. Sci. Instrum 7 (1936) 479-81; 482-8; 8 (1937) $318-9$; 10 (1939) 373-81. 